COMUNICAÇÃO CIENTÍFICA

\title{
CARACTERIZAÇÃO FENOLÓGICA DE PEREIRAS 'HOUSUI' E 'KOUSUI' CULTIVADAS SOB CLIMA SEMIÁRIDO NO NORDESTE DO BRASIL' ${ }^{1}$
}

\author{
PAULO ROBERTO COELHO LOPES ${ }^{2}$, INEZ VILAR DE MORAIS OLIVEIRA ${ }^{3}$, \\ RAISSA RACHEL SALUSTRIANO DA SILVA-MATOS ${ }^{4}$, \\ ÍTALO HERBERT LUCENA CAVALCANTE ${ }^{5}$
}

RESUMO - Observações fenológicas fornecem informações sensíveis para a determinação de como as espécies de plantas respondem às condições climáticas regionais, visando à produção de frutas em diferentes regiões. Assim, o experimento foi conduzido de setembro de 2010 a janeiro de 2011, com o objetivo de caracterizar os estádios fenológicos de pereiras 'Housui' e 'Kousui', cultivadas em clima semiárido do Nordeste do Brasil. Os dados fenológicos (fases) foram coletados no pomar, em observações diárias, desde a aplicação de cianamida hidrogenada (para a quebra de dormência) até o amadurecimento das frutas. Concluíu-se que, em clima semiárido, as pereiras 'Housui' e 'Kousui' concluem seus ciclos fenológicos em 128 dias e 115 dias, respectivamente. Novas pesquisas e mais alguns anos de avaliação serão necessários para gerar um sistema de produção de peras no semiárido nordestino.

Termos para indexação: Pyrus communis L.; requerimento de frio, floração.

\section{PHENOLOGICAL CHARACTERIZATION OF PEAR TREES 'HOUSUI' AND 'KOUSUI' UNDER SEMIARID CONDITIONS IN THE NORTHEASTERN BRAZIL}

\begin{abstract}
Phenological observations provide sensitive information to determine how plant species respond to regional climate conditions in order to produce fruits in different regions. The experiment was conducted from September 2010 to January 2011 with the aim of characterizing the growth stages of pear 'Housui' and 'Kousui', grown in semiarid climate of Northeast Brazil. The phenological data (phases) were collected in the orchard on daily observations from application of hydrogen cyanamide (to break dormancy) until the ripening fruits. It was concluded that in the semiarid climate pear 'Housui' and 'Kousui' they conclude their phenological cycles in 128 days and 115 days, respectively. New research and evaluation over several years will be needed to generate a system of production of pears in the semiarid northeast.
\end{abstract}

Index terms: Pyrus communis L., chilling requirement, flowering.

A pereira pertence à família Rosaceae, que compreende mais de vinte espécies, todas provenientes da Europa e Ásia. No Brasil, a cultura foi introduzida no Sul, região onde até hoje a cultura é predominante. Atualmente, no País, pereiras do tipo europeia (Pyrus communis L.), japonesa [Pyrus pyrifolia (Burn). Nak.] e chinesa (Pyrus bretschneideri Rehd.) são utilizadas como cultivares-copa, enquanto a Pyrus calleryana (Dcne.) é a mais empregada como porta-enxerto (PASA et al., 2011).

No Brasil, as pereiras japonesas das cultivares
'Housui', 'Nijisseiki' e 'Kousui' são as mais cultivadas e possuem alta qualidade comercial; no entanto, são produzidas em pequena escala. A 'Housui' possui polpa crocante, doce, sem adstringência e muito suculenta, os frutos são arredondados, com coloração marrom-dourada quando maduros, apresentam tamanho de médio a grande, tendo boa aceitação no mercado. A 'Kousui' tem coloração marrom-dourada quando maduros, a polpa é doce, crocante e macia, sem adstringência e muito suculenta, apresenta boa produtividade, mas seus frutos são pequenos e de

\footnotetext{
${ }^{1}$ (Trabalho 245-12). Recebido em: 13-09-2012. Aceito para publicação em: 26-04-2013.

${ }^{2}$ Eng. Agr., Dr., Empresa Brasileira de Pesquisa Agropecuária, Embrapa Tropical Semiárido. E-mail: proberto@cpatsa.embrapa.br ${ }^{3}$ Eng $^{\text {a }}$. Agr., Doutora em Produção Vegetal. E-mail: inezvilar@yahoo.com

${ }^{4}$ Bióloga, Bolsista de Mestrado do CNPq, Programa de Solos e Nutrição de Plantas, Universidade Federal do Piauí, Campus Professora Cinobelina Elvas. E-mail: raissasalustriano@yahoo.com.br

${ }^{5}$ Eng. Agr., Dr., Departamento de Agronomia, Universidade Federal do Piauí, Campus Professora Cinobelina Elvas - E-mail: italohlc@ ufpi.edu.br
} 
menor valor comercial que os da 'Housui' (FAORO; ORTH, 2010).

Soster e Latorre (2007) relatam que o conhecimento acerca da fenologia é imprescindível para a escolha da cultivar polinizadora que apresenta floração simultânea à cultivar produtora, definindo, assim, combinações de cultivares que proporcionem maiores rendimentos. Segundo Jackson (2003), para a obtenção de bons índices de frutificação, faz-se necessário superar a autoincompatibilidade por meio da polinização cruzada.

Por muitos séculos, tem havido uma conscientização que há correlação entre a duração dos estádios fenológicos e os eventos climáticos (temperatura, fotoperíodo e precipitação), sendo o estudo dessa relação conhecido como fenologia (AONO; KAZUI, 2008). O estudo da duração das fases da escala fenológica também é essencial para a adoção de técnicas agronômicas adequadas, tais como o momento para a realização da poda, adubação e outros tratos culturais (HISSANO et al., 1990) que, de acordo com Valentini et al. (2001), são imprescindíveis para obteção de resultados satisfatórios de produção.

O presente trabalho foi desenvolvido com o objetivo de caracterizar os estádios fenológicos das pereiras 'Housui' e 'Kousui', e verificar a frutificação na região do Submédio do Vale do São Francisco, em Petrolina-PE.

O estudo foi conduzido de 11-09-2010 a 18-01-2011, em pomar experimental localizado na Estação Experimental de Bebedouro, pertencente à Empresa Brasileira de Pesquisa Agropecuária (Embrapa Semiárido), em Petrolina-PE ( $9^{\circ} 09$ ' $\mathrm{S}, 40^{\circ} 22^{\prime}$ $\mathrm{S}$ e a uma altitude de 365,5 metros acima do nível do mar). De acordo com Köeppen (1948), o clima da região é classificado como Bswh, correspondente a uma região semiárida, com temperatura média anual de $26,0{ }^{\circ} \mathrm{C}$ e mínima e máxima de $21,2{ }^{\circ} \mathrm{C}$ e 32,7 ${ }^{\circ} \mathrm{C}$, respectivamente. A precipitação média anual é de 481,7 milímetros, sendo a maior pluviosidade observada durante os meses de fevereiro a abril. A estação seca ocorre de junho a novembro, e a umidade relativa do ar média é de $67 \%$.

Durante a execução do experimento, os dados climáticos (Figura 1) foram coletados na estação meteorológica automática, localizada na Estação Experimental de Bebedouro.

O pomar de pereiras (Pyrus communis L.) utilizado neste estudo teve o plantio realizado com mudas do tipo raiz nua das cvs. 'Housui' e 'Kousui', enxertadas em 'Pirus' (Pyrus calleryana L.). As mudas foram plantadas em janeiro de 2008 e conduzidas em sistema de 'líder central', sendo as adubações realizadas com base em análise de solo, e os tratamentos fitossanitários foram aplicados quando necessários, assim como o controle de plantas daninhas, e todos os referidos tratos culturais seguiram a recomendação de Quezada et al. (2003).

O pomar é distribuído em fileira, com espaçamento de plantio de 5,0 m entre linhas e 2,0 $\mathrm{m}$ entre plantas, e o sistema de irrigação utilizado foi o de gotejamento com linhas duplas, com dez emissores (fluxo de $2 \mathrm{~L} / \mathrm{h}$ ) por planta. O solo da área de estudo é classificado como ARGISSOLO Amarelo distrófico, textura média/argilosa (GIONGO et al., 2011).

No primeiro ano, foi realizada poda de formação; no segundo ano, antes da desfolha, foi feita poda de condução e arqueamento dos ramos para estimular o desenvolvimento das gemas. A desfolha das pereiras foi realizada em 18 de agosto de 2010, seguida de uma poda para a eliminação do excesso de ramos. A quebra de dormência foi feita com a aplicação de cianamida hidrogenada $\left(\right.$ Dormex $\left.^{\circledR}\right)$ a $1,0 \%$, associada ao óleo mineral $\left(\right.$ Assist $\left.^{\circledR}\right)$ a 2,0\% $\left(1^{\circ}\right.$ de setembro de 2010), aplicados com pulverizador costal, quando as plantas estavam no estádio de gemas dormentes, preconizado por Chabchoub et al. (2010). Para a condução do trabalho, foram selecionadas cinco plantas, das quais foram escolhidos aleatoriamente quatro ramos em cada, os quais foram acompanhados diariamente de 11-09-2010 a 18-01-2011.

O comportamento fenológico das variedades 'Housui' e 'Kousui' foi avaliado através de observações visuais realizadas diariamente, a partir da poda e aplicação de cianamida hidrogenada (Dormex $^{\mathbb{R}}$ ) até a colheita. As determinações dos estádios fenológicos foram baseadas na escala de gemas dormentes de macieira (Malus domestica) de Lopes et al. (2012): A: gema inchada; B: brotação sem folhas; C: brotação com folhas; D: botão branco; $\mathrm{E}$ : início de floração; F: plena floração; G: queda de pétalas, H: frutificação; I: fruto verde em desenvolvimento; J: fruto maduro (Figura 2). A duração de cada estádio fenológico foi registrada em número de dias.

As fases fenológicas foram expressas em percentual, assim como a frutificação. Os dados climáticos e de fases fenológicas foram submetidos a análises de correlação simples realizadas com o Programa Assistat ${ }^{\circledR}$ (SILVA; AZEVEDO, 2009).

A variedade 'Housui' apresentou um ciclo fenológico de 128 dias, no qual foram necessários 13 dias para que as gemas saíssem do estádio de dormência até o estádio de plena floração. A variedade 'Kousui' teve um comportamento precoce em relação à 'Housui', com um ciclo fenológico de 115 dias, dos quais 18 foram necessários para alcançar o estádio de plena floração (Tabela 1; Figura 2).

A rápida floração da 'Housui' em relação à 'Kossui' pode ser explicada pela baixa exigência em 
frio da cv. 'Housui' (Tabela 1), pois segundo Petri et al. (2008), em regiões em que as horas de frio não são satisfeitas, ocorrem variações do período de floração, permitindo inferir que ,provavelmente, a variação no número de dias para a conclusão do ciclo e da floração pode estar relacionada à temperatura. Porém, como as duas cultivares foram submetidas às mesmas condições, o que provavelmente prevaleceu foi o requerimento térmico. Essa grande diferença entre a brotação e o início da floração deve ser vista com cuidado, uma vez que pode trazer prejuízos à frutificação devido, provavelmente, à competição por nutrientes entre o crescimento vegetativo e as gemas floríferas.

Por outro lado, para a cv. 'Housui', a fase fenológica de maior duração correspondeu à fase de frutificação $(\mathrm{H})$ e à fase de frutos verdes em desenvolvimento (I) para o qual foram necessários 62 dias, alcançando um índice de $8 \%$ de frutificação. Para a 'Kousui' foram necessários apenas 50 dias para passar da fase $\mathrm{H}$ para a I na escala fenológica da referida variedade, alcançando um índice de $15 \%$ de pegamento (Tabela 1).

Para ambas as cultivares, os estádios fenológicos correlacionaram-se apenas com a radiação e a precipitação (Tabelas 2 e 3). Dados divergentes são obtidos por Guédon e Legave (2008), nos quais a fenologia da pereira é influenciada principalmente pela temperatura do ar. Chmielewski et al. (2004), estudando na Alemanha outra cultura de clima temperado, a macieira, observaram que todos os estádios fenológicos se correlacionaram com a temperatura, indicando que temperaturas altas, diretamente após a quebra da dormência, podem acelerar os processos de desenvolvimento e, finalmente, levar ao avanço do ciclo fenológico.

Um levatamento realizado com 46 espécies mostrou que $53 \%$ tiveram pelo menos um estádio fenológico impulsionado por mudanças na precipitação, temperatura mínima, ou ambos, principalmente em algumas culturas, tais como a pereira (CHAMBERS; KEATLEY, 2010), resultado convergente ao do presente trabalho, no qual a precipitação apresenta correlação de 0,66 e 0,73 com os estádios fenológicos, correlação considerada por Richman et al. (1980) como moderada, ou seja, dentro da faixa de 0,6 a 0,79 .

Pode-se concluir que, sob condições semiáridas, as pereiras 'Housui' e 'Kousui' apresentam ciclo fenológico de 128 e 115 dias, respectivamente. Mais estudos no decorrer dos anos serão necessários para acompanhar mais ciclos fenológicos, o que permitirá definir os períodos para realização de tratos culturais, possibilitando assim gerar um sistema de produção de peras sob condições semiáridas.

TABELA 1- Duração e percentual dos estádios fenológicos de pereira das cultivares 'Housui' e 'Kousui’ no período de setembro de 2010 a janeiro de 2011. Petrolina-PE, 2010-2011.

\begin{tabular}{ccccc}
\hline \multirow{2}{*}{ Estádios Fenológicos } & \multicolumn{2}{c}{ 'Housui' } & \multicolumn{2}{c}{ 'Kousui' } \\
\cline { 2 - 5 } & Dias & Gemas (\%) & Dias & Gemas $(\%)$ \\
\hline A & 1 & 91 & 1 & 90 \\
B & 6 & 65 & 8 & 53 \\
C & 7 & 50 & 11 & 30 \\
D & 9 & 30 & 13 & 24 \\
E & 10 & 29 & 15 & 23 \\
F & 13 & 28 & 18 & 22 \\
G & 19 & 22 & 24 & 22 \\
H & 25 & 8 & 30 & 15 \\
I & 87 & 8 & 81 & 13 \\
J & 128 & 6 & 115 & 10 \\
\hline
\end{tabular}

A: gema inchada; B: brotação sem folhas; C: brotação com folhas; D: botão branco; E : início de floração; F: plena floração; G: queda de pétalas, H: frutificação; I: fruto verde em desenvolvimento; J: fruto maduro. 


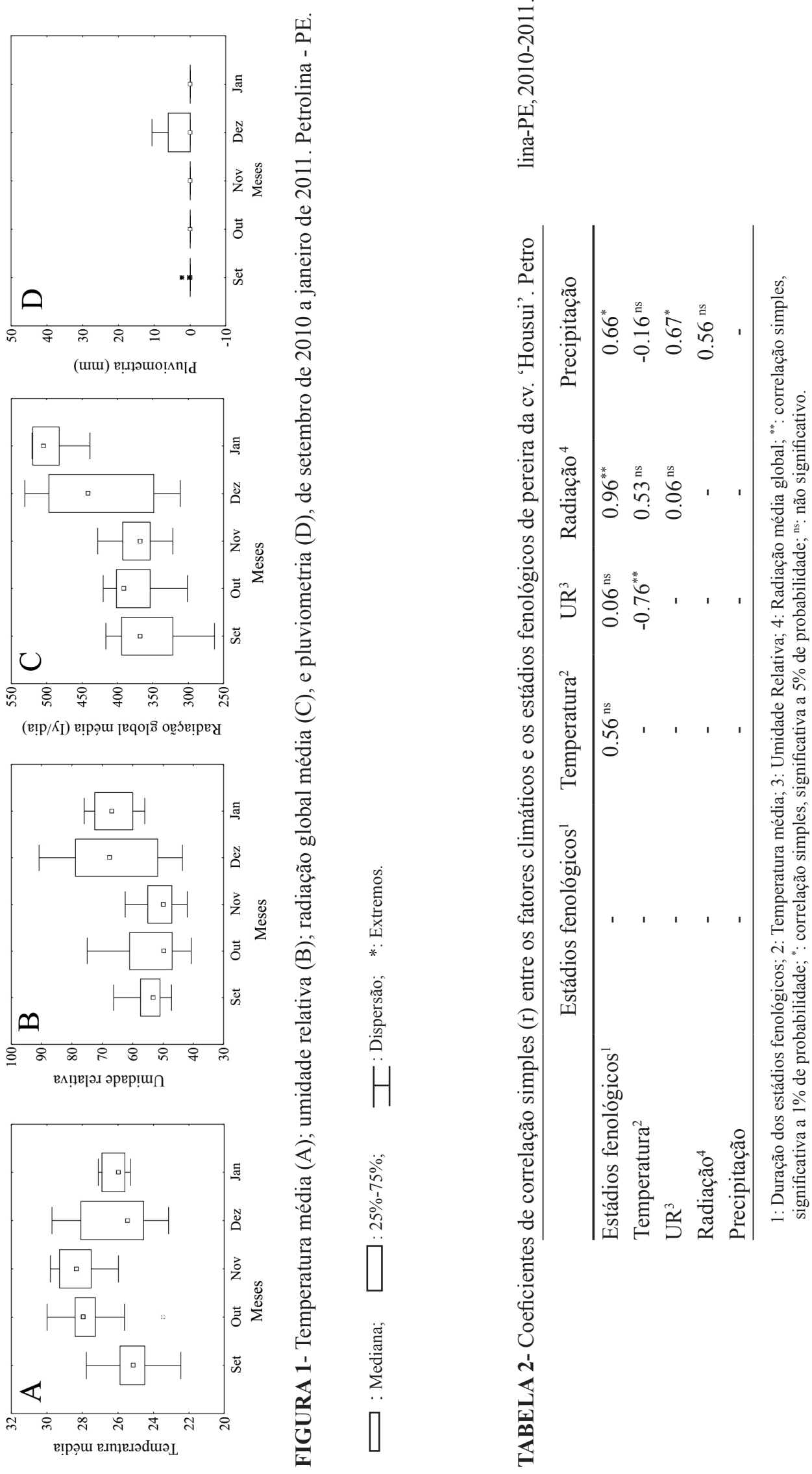


TABELA 3 - Coeficientes de correlação simples (r) entre os fatores climáticos e os estádios fenológicos de pereira da cv. 'Kousui'. Petrolina-PE, 2010-2011.

\begin{tabular}{lccccc}
\hline & Estádios fenológicos $^{1}$ & Temperatura $^{2}$ & $\mathrm{UR}^{3}$ & Radiação $^{4}$ & Precipitação $^{\text {nas }}$ \\
\hline Estádios fenológicos $^{1}$ & - & $0.35^{\text {ns }}$ & $0.27^{\text {ns }}$ & $0.96^{* *}$ & $0.73^{*}$ \\
Temperatura $^{2}$ & - & - & $-0.79^{* *}$ & $0.41^{\text {ns }}$ & $-0.24^{\text {ns }}$ \\
UR $^{3}$ & - & - & - & $0.19^{\text {ns }}$ & $0.72^{*}$ \\
Radiação $^{4}$ & - & - & - & - & 0.59 ns \\
Precipitação & - & - & - & - & - \\
\hline
\end{tabular}

1: Duração dos estádios fenológicos; 2: Temperatura média; 3: Umidade Relativa; 4: Radiação média global; **: correlação simples, significativa a $1 \%$ de probabilidade; ": correlação simples, significativa a $5 \%$ de probabilidade; ns: não significativo.

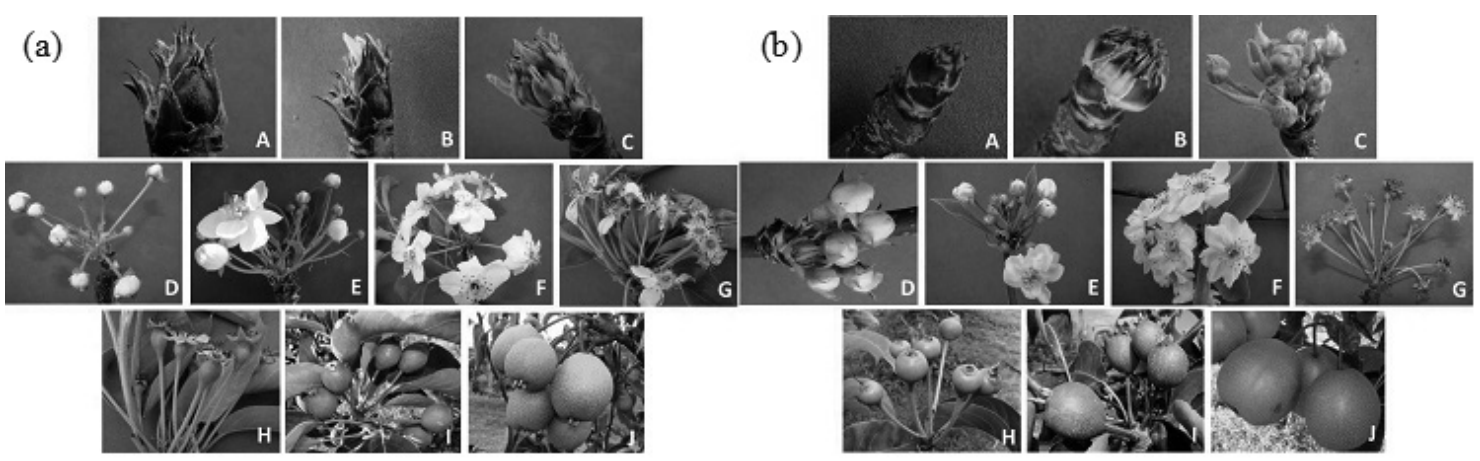

FIGURA 2- Estádios fenológicos das pereira 'Housui' (a) e 'Kousui' (b). Petrolina-PE, 2010-2011. A: gema inchada; B: brotação sem folhas; C: brotação com folhas; D: botão branco; E: início de floração; F: plena floração; G: queda de pétalas, H: frutificação; I: fruto verde em desenvolvimento; J: fruto maduro.

\section{REFERÊNCIAS}

AONO, Y.; KAZUI, K. Phenological data series of cherry tree flowering in Kyoto, Japan, and its application to reconstruction of springtime temperatures since the 9th century. International Journal of Climatology, New York, v. 28, p. 905914, 2008.

CHABCHOUB, M. A.; AOUNALLAH, M. K.; SAHLI, A. Effect of hydrogen cyanamide on bud break, flowering and fruit growth of two pear cultivars (Pyrus communis) under tunisian condition. Acta Horticulturae, Leuven, v. 884, p. 427-431, 2010.

CHAMBERS, L. E.; KEATLEY, M. R. Phenology and climate - early Australian botanical records. Australian Journal of Botany, Melbourne, v. 5 8, p. 473-484, 2010.
CHMIELEWSKI, F.-M.; MÜLLER, A.; BRUNS, E.; Climate changes and trends in phenology of fruit trees and field crops in Germany, 1961-2000. Agricultural and Forest Meteorology, Amsterdam, v. 121, p. 69-78, 2004.

FAORO, I. D; ORTH, A. I. Qualidade de frutos da pereira-japonesa colhidos em duas regiões de Santa Catarina, Brasil. Revista Brasileira de Fruticultura, Jaboticabal, v. 32, n. 1, p. 308-315, 2010.

GIONGO, V.; GALVÃO, S.R. da S.; MENDES, A.M.S.; GAVA, C.A.T.; CUNHA, T.J.F. Soil organic carbon in the brazilian semi-arid tropics. Dynamic Soil, Dynamic Plant, Tokyo, v. 5, n.1, p. 12-20, 2011.

GUÉDON, Y.; LEGAVE, J.M. Analyzing the timecourse variation of apple and pear tree dates of flowering stages in the global warming context. Ecological Modelling, Amsterdam, v. 219, p. 189199, 2008. 
JACKSON, J.E.; Flowers and fruits. In: JACKSON, J.E.; Biology of apples and pears. Cambridge: Cambridge University Press, 2003, p. 368-340.

LOPES, P. R. C.; OLIVEIRA, I. V. de M.; SILVA, R. R. S. da; CAVALCANTE, Í. H. L. Growing Princesa apples under semiarid conditions in northeastern Brazil. Acta Scientiarum. Agronomy, Maringá, v. 35, n. 1, 2013.

PASA, M. da S.; FACHINELLO, J. C.; SCHMITZ, J. D.; SOUZA, A. L. K. de; HERTER, F. G. Hábito de frutificação e produção de pereiras sobre diferentes porta-enxertos. Pesquisa Agropecuária Brasileira, Brasília, v. 46, n. 9, p. 998-1005, 2011.

PETRI, J. L.; HAWERROTH, F. J.; LEITE, G. B. Fenologia de espécies silvestres de macieira como polinizadora das cultivares Gala e Fuji. Revista Brasileira de Fruticultura, Jaboticabal, v. 30, n. 4, p. 868-874, 2008.

QUEZADA, A. C.; NAKASU, B. H.; HERTER, F. G. Pêra produção. Brasília: Embrapa Informação Tecnológica, 2003. 105 p.
RICHMAN, J., MACKRIDES, L.; PRINCE, B. Research methodology and applied Statictic: measurement procedures in research. Physiotherapy Canada, Toronto, v. 32, p. 253-257, 1980.

SILVA, F. de A. S.; AZEVEDO, C. A.V. de. Principal Components Analysis in the Software AssistatStatistical Attendance. In: WORLD CONGRESS ON COMPUTERS IN AGRICULTURE, 7., 2009, Reno. Proceedings... Reno: American Society of Agricultural and Biological Engineers, 2009.

SOSTER, M.T.B.; LATORRE, A.F.; Avaliação da fenologia das cultivares de macieira Imperatriz, Imperial 'Gala' e 'Fuji' em pomar em Bom RetiroSC. Biotemas, Florianópolis, v. 20, n. 4, p. 35-40, 2007.

VALENTINI, N.; ME, G.; FERRERO, R.; SPANNA, F. Use of bioclimatic indexes to characterize phonological phases of apple varieties in Northern Italy. International Journal of Biometeorology, New York, v. 45, p. 191-195, 2001. 